\title{
Some Rearrangement Inequalities on Space of Homogeneous Type
}

\section{Tiejun Chen}

Yiyang Medical College Hunan Pro of China, Yiyang, China

Email: cwwlove@sina.com

Received 9 June 2014; revised 22 July 2014; accepted 2 August 2014

Copyright (C) 2014 by author and Scientific Research Publishing Inc.

This work is licensed under the Creative Commons Attribution International License (CC BY).

http://creativecommons.org/licenses/by/4.0/

c) (i) Open Access

\section{Abstract}

Let $\omega$ be a $A_{\infty}$ Muckenhoupt weight. In this paper we get the estimate of rearrangement $f_{\omega}^{*}$ in homogeneous space that is $f_{\omega}^{*}(t) \leq 2\left(M_{\lambda}^{\#} f\right)_{\omega}^{*}(2 t)+f_{\omega}^{*}(2 t) \quad(0<t<\infty)$. The similar estimate is obtained only on space of $R^{n}$.

\section{Keywords}

Rearrangement, Homogeneous Space, $A_{\infty}$ Weight

\section{Introduction}

We first recall some basic notions about the homogeneous space and the weights we are going to use.

Definition 1 [1]. (Homogeneous space $X$ ). Let $X$ be a set. A function $d: X \times X \rightarrow[0, \infty$ ) is called a quasidistance on $X$ if the following conditions are satisfied:

1) for every $x$ and $y$ in $X, d(x, y) \geq 0$, and $d(x, y)=0$ if and only if $x=y$,

2) for every $x$ and $y$ in $X, d(x, y)=d(y, x)$,

3) there exists a constant $K$ such that $d(x, y) \leq K(d(x, z)+d(z, y))$ for every $x, y$ and $z$ in $X$.

Let $\mu$ be a positive measure on the $\sigma$-algebra of subsets of $X$ generated by the $d$-balls $B(x, r)=\{y: d(x, y)<r\}$, with $x \in X$ and $r>0$. Then a structure $(X, d, \mu)$, with $d$ and $\mu$ as above, is called a space of homogeneous type.

We say that $(X, d, \mu)$ is a space of homogeneous type regular in measure if $\mu$ is regular, that is for every measurable set $E$, given $\varepsilon>0$, there exists an open set $G$ such that $E \subset G$ and $\mu(G-E)<\varepsilon$. In what follows we always assume that the space $(X, d, \mu)$ is regular in measure.

A non-negative locally integrable on homogeneous space $X$ function $\omega(x)$ is called a weight. With any 
weight function we call the measure $\omega(E)=\int_{E} \omega(x) \mathrm{d} x$. Given a measurable function $f$ on homogeneous space $X$, define its non-increasing rearrangement $f_{\omega}^{*}$ with respect to a weight $\omega$ similar to (see [1], p. 32).

$$
f_{\omega}^{*}(t)=\sup _{\omega(E)=t} \inf |f(x)| \quad\left(0<t<\omega\left(R^{n}\right)\right) .
$$

Definition 2 ( $A_{\infty}$ weight) [2]. A weight $\omega$ is in Muckenhoupt's class $A_{\infty}$ respect to $\mu$ if there are positive constants $C$ and $\varepsilon$ such that the inequality:

$$
\frac{\omega(E)}{\omega(B)} \leq C\left(\frac{\mu(E)}{\mu(B)}\right)^{\varepsilon}
$$

holds for every ball $B$ and every measurable set $E \subset B$. The infimum of such $C$ will be denoted by $[\omega] A_{\infty}$.

\section{Basic Lemmas}

Denote doubling condition $D$, a weight $\omega \in D$ if and only if for any ball holds $\omega(2 B) \leq C_{2} \omega(B)$. Clearly if $\omega \in A_{\infty}$ then $\omega \in D$.

Lemma 1 [3]. Let $(X, d, \mu)$ be a space of homogeneous type. Let $\mathrm{B}=\left\{B_{\alpha}: \alpha \in \Gamma\right\}$ be a family of balls in $X$ such that $E=\bigcup_{\alpha \in \Gamma} B_{\alpha}$ is measurable and $\mu(E)<\infty$. Then there exists a disjoint sequence $\left\{B\left(x_{i}, r_{i}\right)\right\} \subset \mathrm{B}$, possibly finite, such that $E \subset \bigcup_{i=1} B\left(x_{i}, C r_{i}\right)$ for some constant $C$. Moreover, every $B \in \mathrm{B}$ is contained in some $B\left(x_{i}, C r_{i}\right)$.

Lemma 2. (C-Z decomposition) [4] [5]. Let $(X, d, \mu)$ be a space of homogeneous type such that the open balls are open sets. Let $\mathrm{f}$ be a nonnegative integrable function defined on $X$, then for every $\lambda \geq m_{X}(f) \quad\left(m_{X}(f)=0\right.$ if $\omega(X)=\infty)$, there exist a sequence of disjoint balls $B_{i}=B\left(x_{i}, r_{i}\right)$ such that if $B_{i}=B\left(x_{i}, C r_{i}\right), C$ is the constant in Lemma [1] then

1) $m_{\overparen{B(f)}} \leq \lambda<m_{B_{i}}(f)$,

2) $m_{\overline{B(f)}} \leq \lambda$ for every ball $B$ centered at $x \in X \backslash \bigcup_{i} \widetilde{B}_{i}$, holds $m_{B}(f) \leq \lambda$.

Lemma 3. $\omega \in D$ and $0<\lambda<1$, If $X$ is a ball and $E \subset X$ is an arbitrary measurable set of positive measure with $\omega(E) \quad \lambda \omega(X)$, there exist mutually disjoint balls $\left\{B_{i}\right\} \subset X$ such that

$B_{i}$ cover $E$ and

$$
\omega\left(E \cap B_{i}\right) / \omega\left(B_{i}\right)>S \geq \omega\left(E \cap \widetilde{B}_{i}\right) / \omega\left(\widetilde{B}_{i}\right) .
$$

Proof: If

$$
\lambda \geq m x(f)=\frac{1}{\omega(X)} \int_{X} f(x) \omega(x) \mathrm{d} \mu(x) .
$$

Letting $f(x)=\chi E(x)$, then

$$
m_{\widetilde{B}_{i}}(\chi E) \leq \lambda<m B_{i}(\chi E)
$$

then

$$
\omega\left(E \cap B_{i}\right) / \omega\left(B_{i}\right)>S \geq \omega\left(E \cap \widetilde{B}_{i}\right) / \omega\left(\widetilde{B}_{i}\right) .
$$

For every ball $B$ centered at $x \in X \backslash U_{i} \widetilde{B_{i}}$

$$
m_{B}(\chi E) \leq \lambda
$$

i.e.

$$
\begin{gathered}
\frac{1}{\omega(B)} \int_{B} \chi E(x) \omega(x) \mathrm{d} u(x) \leq \lambda, \\
\omega(E \cap B) / \omega(B) \leq \lambda .
\end{gathered}
$$


If $E \subset \bigcup_{i} \widetilde{B}_{i}$ there exist $x_{0} \in E$ and $x_{0} \in X-\widetilde{B}_{i}$, now exists $r_{0}$ such that $B\left(x_{0}, r_{0}\right) \subset E$, then

$$
\frac{\omega\left(B\left(x_{0}, r_{0}\right) \cap E\right)}{\omega\left(B\left(x_{0}, r_{0}\right)\right)}=1 \leq \lambda \text {, }
$$

this is a contradiction.

Then $E \subset U_{i} \widetilde{B_{i}}$ and

$$
\lambda \omega\left(B_{i}\right)<\omega\left(E \cap B_{i}\right), \omega\left(E \cap \widetilde{B}_{i}\right) \leq \lambda \omega\left(\widetilde{B}_{i}\right) .
$$

\section{Inequalities Conclusion}

Theorem 1. $\omega \in A_{\infty}, f \geq 0, f \in L_{0}^{1}(X)$, then $f_{\omega}^{*}(t) \leq 2\left(M_{\lambda \omega}^{\#} f\right)_{\omega}^{*}(2 t)+f_{\omega}^{*}(2 t) \quad\left(0<t<\omega(X) / 5 C_{1}\right)$.

Proof: The proof is similar to Lerner [5]-[7],

$$
\begin{gathered}
|C| \leq \inf _{\chi \in B}(|f-C|+|f|)_{\omega}^{*}(\omega(B)) \leq\left((f-C)_{\chi B}\right)_{\omega}^{*}(\lambda \omega(B))+\left(f \chi_{B}\right)_{\omega}^{*}((1-\lambda) \omega(B)) . \\
\left(f \chi_{B}\right)_{\omega}^{*}(\lambda \omega(B)) \leq\left((f-C) \chi_{B}\right)_{\omega}^{*}(\lambda \omega(B))+|C| \leq 2\left((f-C) \chi_{B}\right)_{\omega}^{*}(\lambda \omega(B))+\left(f \chi_{B}\right)_{\omega}^{*}((1-\lambda) \omega(B)) .
\end{gathered}
$$

From [6], We get two collections of balls $\left\{B_{i}^{s}: i \in N\right.$ with $\left.s=\lambda, \lambda / 2, \cdots\right\}$, then

$$
\omega\left(E \cap B_{i}^{s}\right) / \omega\left(B_{i}^{s}\right)>S \geq \omega\left(E \cap \widetilde{B_{i}^{s}}\right) / \omega\left(\widetilde{B_{i}^{s}}\right) .
$$

Fix $X$, with $0<t \leq \frac{1}{5 C_{2}} \omega(X), \lambda<\frac{1}{5 C_{2}}$, for all $E, \omega(E)=t$ there is $\omega(E) \leq \frac{1}{5 C_{2}} \omega(X)$, then exist disjoint balls $\left\{B_{i}^{s} \subset X\right\}$, hold

$$
\omega\left(E \cap B_{i}^{s}\right)>s \omega\left(B_{i}^{s}\right), \quad \omega\left(E \cap \widetilde{B_{i}^{s}}\right)>s \omega\left(\widetilde{B_{i}^{s}}\right) .
$$

Which contains

$$
\omega\left(E \cap B_{i}^{\lambda / 2}\right)>\frac{1}{10 C_{2}} \omega\left(B_{i}^{\lambda / 2}\right), \quad \omega\left(E \cap \widetilde{B_{i}^{\lambda}}\right)>\frac{1}{5 C_{2}} \omega\left(\widetilde{B_{i}^{\lambda}}\right) .
$$

Then

$$
\sum_{i} \omega\left(\widetilde{B_{i}^{\lambda}}\right) \geq \frac{1}{\lambda} \sum_{i} \omega\left(E \cap \widetilde{B_{i}^{\lambda}}\right) \geq 5 C_{2} \sum_{i} \omega\left(E \cap \widetilde{B_{i}^{\lambda}}\right)=5 C_{2} \omega E=5 C_{2} t
$$

Select from $B_{i}^{\lambda}$ the balls $B_{i}^{\prime}, \quad i \in F$ which are not contained in $\Omega$, $\Omega=\left\{x \in X: M_{\lambda \omega}^{\#} f(x)>\left(M_{\lambda \omega}^{\#} f\right)(2 t)\right\}$. That is for all $i \in F, B_{i}^{\prime} \cap \Omega^{c} \neq \phi$. There exist $x_{0} \in B_{i}^{\prime}, x_{0} \in \Omega^{c}$, then

$$
\inf _{x \in B_{i}^{\prime}} M_{\lambda \omega}^{\#} f(x) \leq M_{\lambda \omega}^{\#} f\left(x_{0}\right) \leq\left(M_{\lambda \omega}^{\#} f\right)_{\omega}^{*}(2 t) .
$$

Note that $\omega(\Omega) \leq 2 t$,

$$
\sum_{i} \omega\left(B_{i}^{\prime}\right) \geq \sum_{i} \omega\left(B_{i}\right)-\omega(\Omega) \geq 5 t-3 t=2 t .
$$

Since

$$
U_{i} \widetilde{B_{i}}-\Omega=U_{i}\left(\widetilde{B_{i}}-\Omega\right)=\left(U_{i \in F}\left(\widetilde{B_{i}}-\Omega\right)\right) \cup\left(U_{i \in F^{\prime}}\left(\widetilde{B_{i}}-\Omega\right)\right)=U_{i \in F}\left(\widetilde{B_{i}}-\Omega\right) \subset U_{i \in F} \widetilde{B_{i}} .
$$

Then

$$
\sum_{i} C_{2} \omega\left(B_{i}\right) \geq \sum_{i} \omega\left(\widetilde{B}_{i}\right) \geq 5 C_{2} t
$$

i.e.

$$
\sum_{i} \omega\left(B_{i}\right) \geq 5 t .
$$




$$
\inf _{i}\left(f \cdot \chi B_{i}^{\prime}\right)_{\omega}^{*}\left(\left(1-\frac{1}{5 C_{2}}\right) \omega\left(B_{i}^{\prime}\right)\right) \leq f_{\omega}^{*}\left(1-\frac{1}{5 C_{2}} 3 t\right) \leq f_{\omega}^{*}(2 t) .
$$

We have

$$
\begin{aligned}
\inf _{x \in E}|f(x)| & \leq \inf _{i} \inf _{x \in E \cap B_{i}^{\prime}}|f(x)| \leq \inf _{i}\left(f \chi B_{i}^{\prime}\right)_{\omega}^{*}\left(\omega\left(B_{i}^{\prime} \cap E\right)\right) \\
& \leq \inf _{i}\left(f \chi B_{i}^{\prime}\right)_{\omega}^{*}\left(\omega\left(B_{i}^{\prime}\right) / 5 C_{2}\right) \leq 2\left(M_{\lambda \omega}^{\#} f\right)_{\omega}^{*}(2 t)+f_{\omega}^{*}(2 t) .
\end{aligned}
$$

Taking supremum over all $E \subset X$ with $\omega(E)=t$, we get the argument .

\section{Fund}

A project supported by scientific research fund of Hunan provincial education department in China (NO: 13C955).

\section{References}

[1] Chong, K.M. and Rice, N.M. (1971) Equimeasurable Rearrangements of Functions. Queen’s Papers in Pure and Appl. Math., 28, Queen's University, Kingston.

[2] Aimar, H. (1985) Singular Integrals and Approximate Identities on Spaces of Homogeneous Type. Transactions of the American Mathematical Society, 292,135-153. http://dx.doi.org/10.1090/S0002-9947-1985-0805957-9

[3] Aimar, H. (1991) Rearrangement and Continuity Properties of BMO(`) Functions on Spaces of Homogeneous Type. Annali della Scuola Normale Superiore di Pisa, 4, 353-362.

[4] Lerner, A.K. (1998) On Weighted Estimates of Non-Increasing Rearrangements. East Journal on Approximations, 4, 277-290.

[5] Lerner, A.K. (2004) Weighted Rearrangement Inequalities for Local Sharp Maximal Functions. Transactions of the American Fisheries Society, 357, 2445-2465. http://dx.doi.org/10.1090/S0002-9947-04-03598-6

[6] Lerner, A.K. (2003) On the John-Strömberg Characterization of BMO for Nondoubling Measures. Real Analysis Exchange, 28, 465-474.

[7] Pradolini, G., Salinas, O. and Fe, S. (2007) Commutators of Singular Integrals on Spaces of Homogeneous Type. Czechoslovak Mathematical Journal, 57, 75-93. http://dx.doi.org/10.1007/s10587-007-0045-9 
Scientific Research Publishing (SCIRP) is one of the largest Open Access journal publishers. It is currently publishing more than 200 open access, online, peer-reviewed journals covering a wide range of academic disciplines. SCIRP serves the worldwide academic communities and contributes to the progress and application of science with its publication.

Other selected journals from SCIRP are listed as below. Submit your manuscript to us via either submit@scirp.org or Online Submission Portal.
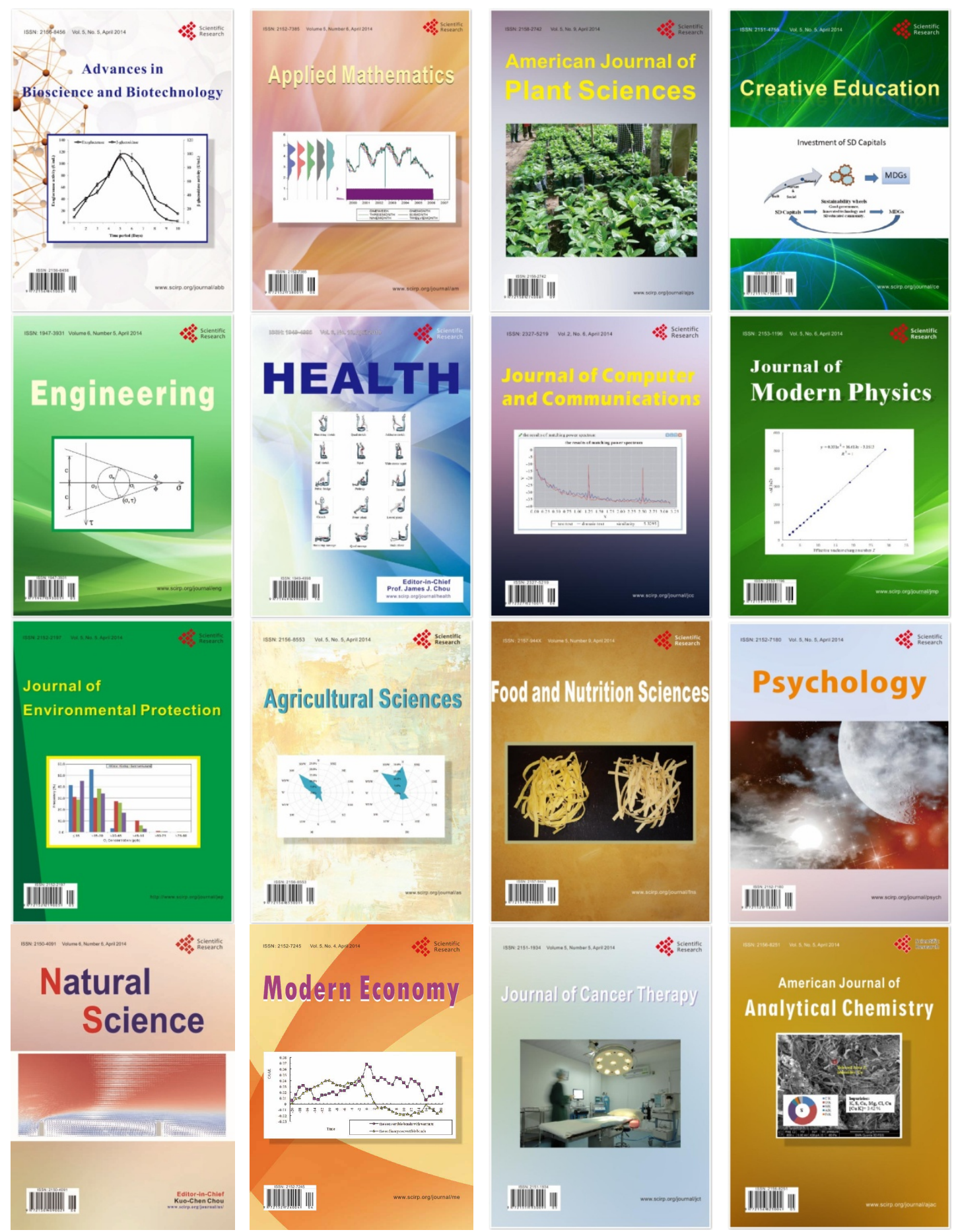